\title{
Nonlinear Adaptive Control of a Two-Vehicle Convoy
}

\author{
P. Petrov
}

Faculty of Mechanical Engineering, Technical University of Sofia, 8, Kl. Ohridski Str., 1797 Sofia, Bulgaria

\begin{abstract}
This paper describes the modeling of a two-vehicle convoy and the design of a vehicle following controller that tracks the trajectory of the vehicle ahead with prescribed inter-vehicle distance. Kinematic equations of the system are formulated applying standard robotic methodology. We consider autonomous vehicle following without any information obtained from road infrastructure or communicated from the lead vehicle. Assuming that the leader linear and angular velocities, as well the curvature radius of the path traveled by the lead vehicle, are unknown constant parameters, an adaptive tracking controller is proposed. With only the current inter-vehicle relative position and orientation available for feedback control, the control velocities of the following vehicle are computed using the leader velocity estimates obtained from the dynamic (adaptive) part of the proposed controller. For constant velocity maneuvers of the leader, at steady state, the two-vehicle convoy will travel concentric arcs of same radii with prescribed inter-vehicle spacing. Various simulation results demonstrating the performance of the controller are included.
\end{abstract}

Keywords: Autonomous vehicle convoy, vehicle following, kinematic model, nonlinear adaptive control.

\section{INTRODUCTION}

In recent years, the problem of automatic vehicle following in a convoy-like fashion, where each vehicle tracks the path taken by the preceding car at a desired separation distance, has attracted a considerable interest. The research effort into vehicle platooning and automatic vehicle following is based on the fact that often multiple vehicles have the possibility of solving transportation problems more efficiently than a single vehicle. This scenario is very useful in the case of military convoys [1-3], convoys of commercial vehicles $[4,5]$, personal vehicles $[6]$ or in the public urban transport $[7,8]$.

Lateral (steering) control and longitudinal (spacing and speed) control were initially designed as two separate control systems. Each controller was designed as if the longitudinal and the steering vehicle dynamics were independent. Much research has been done in the study of longitudinal control of a platoon of vehicles on a straight line with different spacing policies [9]. For the lateral control of a vehicle convoy two basic concepts are studied. In [10], the lateral control is focused on the concept of cooperation between the vehicle and the road. The lane keeping performance relies on suitable reference markers and communication infrastructure to supply the vehicle with information about the road geometry. As an alternative, the concept of autonomous vehicle following [6], implies that the ego-vehicle tracks the trajectory of the vehicle ahead instead of the road by using on-board sensors that detect the vehicle relative position with respect to the lead vehicle. One of the principle issues in designing controllers for autonomous vehicle following is to determine the desired trajectory of the following vehicle.

*Address correspondence to this author at the Faculty of Mechanical Engineering, Technical University of Sofia, 8, Kl. Ohridski Str., 1797 Sofia, Bulgaria; E-mail: ppetrov@tu-sofia.bg
Previous work in generating the desired trajectory for the ego-vehicle has used several approaches including "trajectory-based approach" [11], that uses the time history of the lead vehicle, i.e., the ego-vehicle has to follow the path of the leader, not the lead vehicle itself. In [7], a tracking method involves using a contour of constant curvature to interpolate a trajectory between the following and the lead vehicle. In [12], a direct control of the following vehicle using information from on-board sensing without inter-vehicle communication has been proposed. A different approach to vehicle following control by using inter-vehicle communication has also been used [13]. An interesting solution is the use of RTK GPS sensors, which can provide in real time localization with high accuracy [14]. In combination with inter-vehicle communication, these sensors permit to interchange absolute localization measurements.

In this paper, we consider the problem of autonomous vehicle following without the use of road infrastructure or inter-vehicle communication. The only information the following (robot) vehicle can use for feedback control is the current relative position and orientation with respect to the lead vehicle obtained from onboard sensors, which monitor the rear end of the lead vehicle.

Our contributions in this paper are two-fold: an adaptive nonlinear control of the following vehicle that allows the ego-vehicle to track the trajectory of the vehicle ahead in the presence of unknown leader linear and angular velocities, as well the curvature radius of the path traveled by the lead vehicle, and the use of robotic oriented approach to mathematical leader/follower modeling that allows the following vehicle to track the path taken by the lead vehicle at a specific position in the plan with respect to the leader.

One of the objectives of this paper is to develop a mathematical model of a vehicle convoy suitable for feedback control design. We formulate the kinematic 
equations of a two-vehicle convoy in error coordinates applying standard robotic methodology. One of the challenges in designing controllers for automatic vehicle tracking is to decide on the desired trajectory of the following vehicle. On a curved road section, traveling an arc concentric to that traveled by the lead car but with bigger or smaller radius may be unacceptable from a practical point of view, (for example, the problem of "cutting the corner"). Assuming that the lead vehicle linear and angular velocities are unknown constant parameters, and the curvature radius of the path taken by the lead vehicle is also unknown, we are interested in designing a controller for the following vehicle, such that, at steady state, the following vehicle tracks the path taken by the lead vehicle with prescribed inter-vehicle distance. The approach used in this paper is closely related to that presented in [12] in the sense that it consists in tracking a reference virtual point, which is positioned at a desired known distance behind the lead vehicle with a virtual reference point located at a desired distance ahead of the following vehicle. This approach has been inspired from our previous work [15] concerning the problem of controlling a LHD (Load-Haul-Dump) vehicle. At steady-state, the two linked units of the LHD vehicle with equal unit length will travel concentric arcs of same radii. In this paper, the adaptive control designed is based on a reduced order dynamics and achieves asymptotic stabilization of the closed-loop system in error coordinates. The control velocities (control inputs) of the following vehicle are computed using the leader velocity estimates obtained from the dynamic (adaptive) part of the controller, which was designed as a parameter update law. The stability of the internal dynamics is also analyzed. Throughout this paper, feedback control design and stability analysis are performed via Lyapunov techniques.

The organization of this paper is as follows: in Section II, the development of a nonlinear kinematic model of the vehicle convoy suitable for feedback control is presented. The problem formulation is given in Section III. In Section IV, the adaptive tracking controller of the two-vehicle convoy is proposed. Section V contains simulation results which illustrate the effectiveness of the proposed controller. Conclusions are presented in Section VI.

\section{KINEMATIC MODELING}

\section{A. Vehicle Kinematic Model}

\section{1) Coordinate System Assignments}

In this section, we apply standard robotic nomenclature for translational and rotational displacements and velocities, and methodology [16] to model the kinematics of the vehicle in the convoy. Fig. (1) depicts the schematic of the vehicles considered in this paper. From now on, the index $i=1$ corresponds to the lead vehicle, and $i=2$ corresponds to the following vehicle in the platoon. The vehicle has four nondeformable wheels. The wheels are assumed to roll on a horizontal plane without slipping. The longitudinal base $A_{i} B_{i}$ of the vehicle is denoted by $l_{i},(i=l, 2)$, To simplify the derivation of the vehicle control algorithms, we a use a planar bicycle 2DOFs vehicle model where two virtual wheels are located at the midpoints of the front and rear wheel axles, (Fig. 1). Although these two wheels do not exist, it is assumed that they comply with the wheel rolling without slipping conditions.

To describe the position and orientation of the vehicle in the plane, we assign the following coordinate frames (Fig. 1).

$A_{i} x_{A i} y_{A i}$ : Vehicle coordinate system located at the center of the rear vehicle axle and stationary with respect to the vehicle body; the $x_{A i}$ axis is along the longitudinal base of the vehicle, $(i=1,2)$.

- $\quad B_{i} x_{i} y_{i}$. Wheel coordinate system with origin placed at the center of the front steering wheel; the $x$, axis is in the direction of the wheel orientation, $(i=1,2)$.

- $\quad$ Fxy denotes an inertial coordinate frame in the plane to travel.

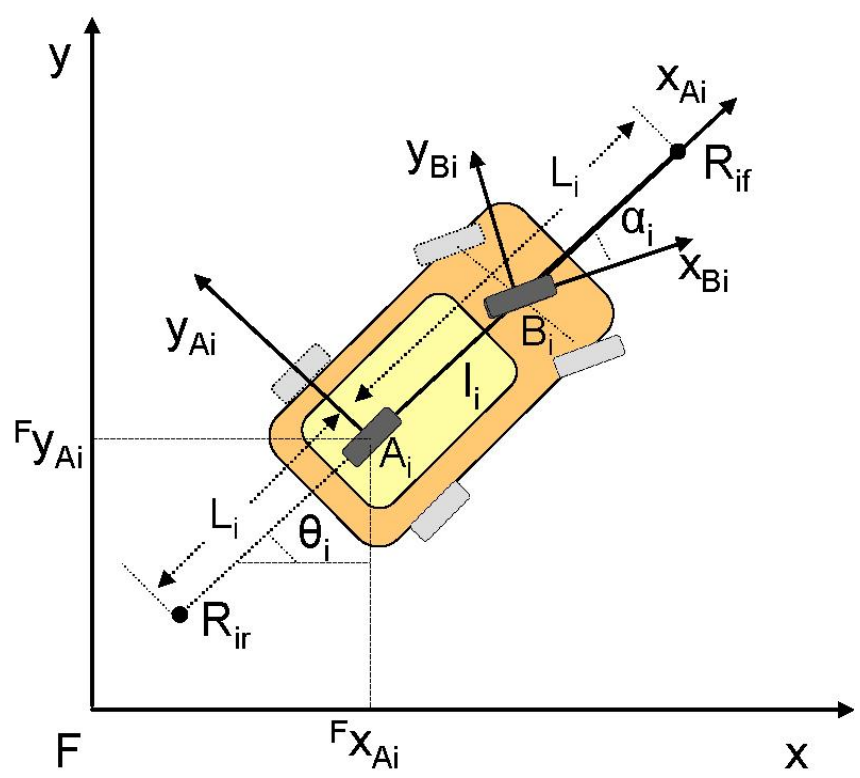

Fig. (1). Schematic of the vehicle.

The coordinates of a reference point $A_{i}$ placed at the center of the rear vehicle axle, with respect to an inertial frame $F x y$, are denoted by $\left({ }^{F} x_{A i},{ }^{F} y_{A i}\right)$. The angle $\theta_{i}$ is the orientation angle of the vehicle with respect to the frame Fxy. The angle $\alpha_{i}$ is the front wheel steering angle. The steering angle is measured with respect to the vehicle body. The reference points $R_{1 r}$ and $R_{2 f}$ located on the longitudinal vehicle axle are associated with the lead and following vehicle, respectively. The point $R_{l r}$ is placed a $L_{l}$ distance behind point $A_{1}$ of the lead vehicle and point $R_{2 f}$ is placed a $L_{2}$ distance in front of point $A_{2}$ of the following vehicle.

Since the vehicle is assumed to move on a planar surface, in what follows, we use $3 \times 3$ rather than $4 \times 4$ homogeneous transformation matrices ${ }^{A} T_{B}$ to transform the coordinates of a point $S$ in coordinate frame $B$ denoted by ${ }^{B} p_{s}$ to its corresponding coordinates ${ }^{A} p_{s}$ in the coordinate frame A.

Using the above notations, the assignment of the coordinate frames results in the following transformation matrices between coordinate systems 


$$
\begin{aligned}
{ }^{A_{i}} T_{B_{i}} & =\left[\begin{array}{ccc}
\cos \alpha_{i} & -\sin \alpha_{i} & l_{i} \\
\sin \alpha_{i} & \cos \alpha_{i} & 0 \\
0 & 0 & 1
\end{array}\right] \\
{ }^{F} T_{A_{i}} & =\left[\begin{array}{ccc}
\cos \theta_{i} & -\sin \theta_{i} & { }^{F} x_{A i} \\
\sin \theta_{i} & \cos \theta_{i} & { }^{F} y_{A i} \\
0 & 0 & 1
\end{array}\right] .
\end{aligned}
$$

The transformation matrices (1) and (2) are applied to determine the position kinematics of the vehicle.

Using (1) and (2), the position of point $B_{i}$ in the inertial frame $F$ is

$$
\begin{aligned}
& { }^{F} p_{B_{i}}={ }^{F} T_{A_{i}}{ }^{A_{i}} T_{B_{i}}{ }^{B_{i}} p_{B_{i}} \\
& =\left[\begin{array}{ccc}
\cos \theta_{i} & -\sin \theta_{i} & { }^{F} x_{A_{i}} \\
\sin \theta_{i} & \cos \theta_{i} & { }^{F} y_{A_{i}} \\
0 & 0 & 1
\end{array}\right]\left[\begin{array}{ccc}
\cos \alpha_{i} & -\sin \alpha_{i} & l_{i} \\
\sin \alpha_{i} & \cos \alpha_{i} & 0 \\
0 & 0 & 1
\end{array}\right]\left[\begin{array}{l}
0 \\
0 \\
1
\end{array}\right] \\
& =\left[\begin{array}{c}
{ }^{F} x_{A_{i}}+l_{i} \cos \theta_{i} \\
{ }^{F} y_{A_{i}}+l_{i} \sin \theta_{i} \\
1
\end{array}\right] .
\end{aligned}
$$

Using (2), the homogeneous coordinates of the rear reference point $R_{I r}$ associated with the lead vehicle in frame Fxy are

${ }^{F} p_{R_{1 r}}={ }^{F} T_{A_{1}}{ }^{A_{1}} p_{R_{1 r}}=\left[\begin{array}{c}{ }^{F} x_{A_{1}}-L_{1} \cos \theta_{1} \\ { }^{F} y_{A_{1}}-L_{1} \sin \theta_{1} \\ 1\end{array}\right]$.

Similarly, the homogeneous coordinates of the front reference point $R_{I r}$ associated with the following vehicle in frame Fxy are

${ }^{F} p_{R_{2 f}}={ }^{F} T_{A_{21}}{ }^{A_{2}} p_{R_{2 r}}=\left[\begin{array}{c}{ }^{F} x_{A_{2}}+L_{2} \cos \theta_{2} \\ { }^{F} y_{A_{2}}+L_{2} \sin \theta_{2} \\ 1\end{array}\right]$.

\section{2) Non-Holonomic Constaraints}

If the rotation of the wheels with respect to their proper axes is ignored, the vehicle configuration can be described by four generalized coordinates $q_{i}=\left[{ }^{F} x_{A i}{ }^{F} y_{A i}, \theta_{i}, \alpha_{i}\right]^{T} \in \mathfrak{R}^{4}$.

Differentiating (3), the components of the velocity of point $B_{i}$ with respect to the inertial frame Fxy and expressed in Fxy are

$$
{ }^{F} \dot{p}_{B_{i}}=\left[\begin{array}{c}
{ }^{F} \dot{x}_{B_{i}} \\
{ }^{F} \dot{y}_{B_{i}} \\
0
\end{array}\right]=\left[\begin{array}{c}
{ }^{F} \dot{x}_{A_{i}}-l_{i} \dot{\theta}_{i} \sin \theta_{i} \\
{ }^{F} \dot{y}_{A_{i}}+l_{i} \dot{\theta}_{i} \cos \theta_{i} \\
0
\end{array}\right] .
$$

In order to derive the non-holonomic constraints of the front virtual wheel, the velocity of point $B_{i}$ relative to frame $F x y$ is expressed in frame $B_{i} x_{i} y_{i}$

$$
\begin{aligned}
& { }^{B_{i}} \dot{p}_{B_{i}}=\left[\begin{array}{c}
{ }^{B_{i}} v_{B_{i} x_{B_{i}}} \\
{ }^{B_{i}} v_{B_{i} y_{B_{i}}} \\
0
\end{array}\right]=\left({ }^{F} T_{A_{i}}{ }^{A_{i}} T_{B_{i}}\right)^{-1 F} \dot{p}_{B_{i}} \\
& =\left[\begin{array}{ccc}
\cos \left(\theta_{i}+\alpha_{i}\right) & \sin \left(\theta_{i}+\alpha_{i}\right) & * \\
-\sin \left(\theta_{i}+\alpha_{i}\right) & \cos \left(\theta_{i}+\alpha_{i}\right) & * \\
0 & 0 & 1
\end{array}\right]\left[\begin{array}{c}
{ }^{F} \dot{x}_{B_{i}} \\
{ }^{F} \dot{y}_{B_{i}} \\
0
\end{array}\right] .
\end{aligned}
$$

where the terms indicated by $(*)$ are irrelevant in the computation. Based on the assumption of rolling without lateral sliding, one has ${ }^{B_{i}} v_{B_{i} y_{B_{i}}}=0$, where ${ }^{B_{i}} v_{B_{i} y_{B_{i}}}$ is the component of the velocity of point $B_{i}$ along the $y_{B i}$ axis of frame $B_{i} x_{B i} y_{B i}$. From the second line of equality (7) and by using expressions (6) for ${ }^{F} \dot{p}_{B_{i}}$, the non- holonomic constraint for the front virtual wheel can be written in the form

$0=-{ }^{F} \dot{x}_{A_{i}} \sin \left(\theta_{i}+\alpha_{i}\right)+{ }^{F} \dot{y}_{A_{i}} \cos \left(\theta_{i}+\alpha_{i}\right)+l_{i} \dot{\theta}_{i} \cos \alpha_{i}$.

Likewise, using (2), the non-holonomic constraint imposed on the rear virtual wheel can be derived from the second line of the following expression

${ }^{A_{i}} \dot{p}_{A_{i}}=\left[\begin{array}{c}{ }^{A_{i}} v_{A_{i} x_{A_{i}}} \\ { }^{A_{i}} v_{A_{i} y_{A_{i}}} \\ 0\end{array}\right]={ }^{F} T_{A_{i}}{ }^{-1 F} \dot{p}_{A_{i}}$

$=\left[\begin{array}{ccc}\cos \theta_{i} & \sin \theta_{i} & * \\ -\sin \theta_{i} & \cos \theta_{i} & * \\ 0 & 0 & 1\end{array}\right]\left[\begin{array}{c}{ }^{F} \dot{x}_{A_{i}} \\ { }^{F} \dot{y}_{A_{i}} \\ 0\end{array}\right]$.

Expressing the fact that the wheel cannot move in lateral direction ${ }^{A_{i}} v_{A_{i} y_{i}}=0$, the non-holonomic constraint is derived from second line of (9) as follows

$0=-{ }^{F} \dot{x}_{A_{i}} \sin \theta_{i}+{ }^{F} \dot{y}_{A_{i}} \cos \theta_{i}$.

Combining equations (8) and (10), the vehicle nonholonomic constraints can be written in the form

$C_{i}(q) \dot{q}_{i}=0$

where $C_{i}\left(q_{i}\right)$ is a $2 x 4$ full rank matrix of the form

$C_{i}(q)=\left[\begin{array}{cccc}-\sin \theta_{i} & \cos \theta_{i} & 0 & 0 \\ -\sin \left(\theta_{i}+\alpha_{i}\right) & \cos \left(\theta_{i}+\alpha_{i}\right) & l_{i} \cos \alpha_{i} & 0\end{array}\right]$

and $\dot{q}_{i}$

$\dot{q}_{i}=\left[\begin{array}{llll}{ }^{F} \dot{x}_{A_{i}} & F \dot{y}_{A_{i}} & \dot{\theta}_{i} & \dot{\alpha}_{i}\end{array}\right]^{T}$

is the vector of generalized velocities. The constraint equation (11) can be converted into an affine driftless control system

$\dot{q}_{i}=D_{i}\left(q_{i}\right) \eta$

where the columns of the $4 \times 2$ matrix $D_{i}\left(q_{i}\right)$ 


$$
D_{i}\left(q_{i}\right)=\left[\begin{array}{cc}
\cos \theta_{i} & 0 \\
\sin \theta_{i} & 0 \\
\frac{\tan \alpha_{i}}{l_{i}} & 0 \\
0 & 1
\end{array}\right]
$$

form a basis of the null space of $C_{i}\left(q_{i}\right)$. The control input $\eta_{i}=\left[{ }^{A_{i}} v_{A_{i} x_{A_{i}}}, \omega_{\alpha i}\right]^{T}$ is a $2 \times 1$ vector of independent quasivelocities which parameterize the degree of freedom of the system, where ${ }^{{ }^{A}} v_{A_{i} x_{A_{i}}}$ is the velocity of point $A_{i}$ (the midpoint of the rear virtual wheel) and $\omega_{o i}$ is the steering angular velocity of the front wheel.

Differentiating (4) and using the first two equations of (15) for $i=1$, a kinematic model of the lead vehicle can be written in the form

$$
{ }^{F} \dot{p}_{R_{1 r}}=\left[\begin{array}{c}
{ }^{F} \dot{x}_{R_{1 r}} \\
{ }^{F} \dot{y}_{R_{1 r}} \\
\dot{\theta}_{1}
\end{array}\right]=\left[\begin{array}{cc}
\cos \theta_{1} & L_{1} \sin \theta_{1} \\
\sin \theta_{1} & L_{1} \cos \theta_{1} \\
0 & 1
\end{array}\right]\left[\begin{array}{c}
{ }^{A_{1}} v_{A_{1} x_{A_{1}}} \\
\omega_{1}
\end{array}\right]
$$

where $\omega_{1}:=\dot{\theta}_{1}$ is the angular velocity of the vehicle.

Similarly, differentiating (5) and using the first two equations of (15) for $i=2$, a kinematic model of the following vehicle is obtained in the form

$$
{ }^{F} \dot{p}_{R_{2 f}}=\left[\begin{array}{c}
{ }^{F} \dot{x}_{R_{2 f}} \\
{ }^{F} \dot{y}_{R_{2 f}} \\
\dot{\theta}_{2}
\end{array}\right]=\left[\begin{array}{cc}
\cos \theta_{2} & -L_{2} \sin \theta_{2} \\
\sin \theta_{2} & L_{2} \cos \theta_{2} \\
0 & 1
\end{array}\right]\left[\begin{array}{c}
{ }_{2}^{A_{2}} v_{A_{2} x_{A_{2}}} \\
\omega_{2}
\end{array}\right]
$$

where $\omega_{2}:=\dot{\theta}_{2}$ is the angular velocity of the vehicle. In this paper, the vehicle angular velocity (the front wheel steering angle $a_{2}$, respectively) is considered as a control input instead of the steering angle velocity. From the third equation of (15) for $i=2$, the front wheel steering angle can be expressed in terms of the vehicle angular velocity as follows

$$
\alpha_{2}=a \tan \left(l_{2} \frac{\dot{\theta}_{2}}{{ }^{A_{2}} v_{A_{2} y_{A_{2}}}}\right) \text {. }
$$

\section{B. Relative Kinematics}

A plan view of a two-vehicle convoy moving on a horizontal plan is shown in Fig. (2).

A virtual reference points $R_{l r}$ associated with the lead vehicle is defined on the longitudinal vehicle axle at a distance $L_{l}$ behind the rear vehicle axle. A virtual reference point $R_{2 f}$ is located on the longitudinal vehicle axle at a distance $L_{2}$ in front of the rear vehicle axle of the following vehicle. Two coordinate systems $R_{l r} x y$ and $R_{2 f} x y$ whose origins are located at the vehicle reference points $R_{l r}$ and $R_{2 f}$, respectively, (the $\mathrm{x}$-axes are oriented along the longitudinal vehicle axles) are defined to describe the relative position and orientation of the vehicles.

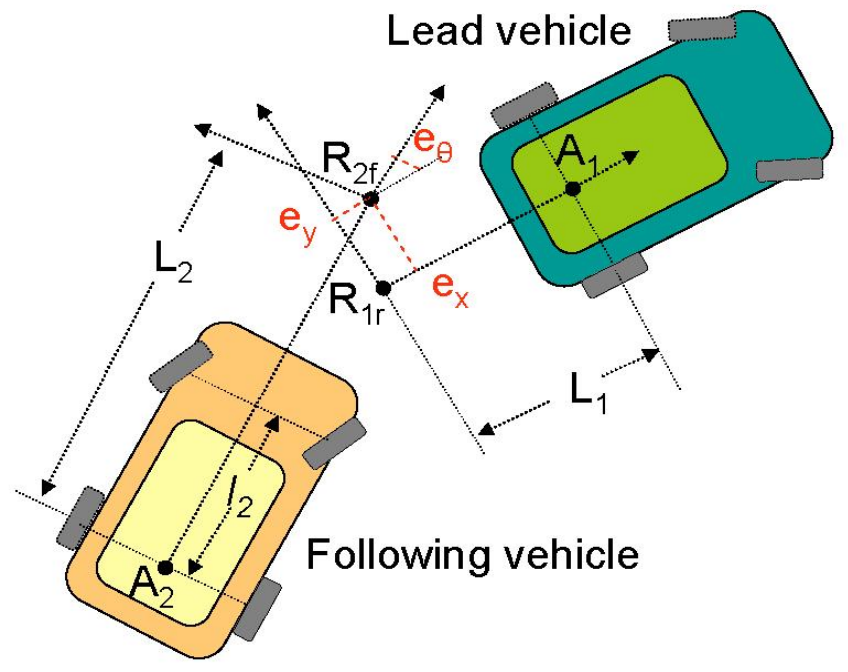

Fig. (2). System configuration for autonomous following.

The coordinates and orientation of the frame $R_{2 f} x y$ in the coordinates frame $R_{l r} x y$ can be expressed as follows (19)

$\left[\begin{array}{l}e_{x} \\ e_{y} \\ e_{\theta}\end{array}\right]=\left[\begin{array}{ccc}\cos \theta_{1} & \sin \theta_{1} & 0 \\ -\sin \theta_{1} & \cos \theta_{1} & 0 \\ 0 & 0 & 1\end{array}\right]\left[\begin{array}{c}{ }^{F} x_{R_{2 f}}-{ }^{F} x_{R_{1 r}} \\ { }^{F} y_{R_{2 f}}-{ }^{F} y_{R_{1 r}} \\ \theta_{2}-\theta_{1}\end{array}\right]$

where $e=\left[e_{x}, e_{y}, e_{\theta}\right]^{T} \in \mathfrak{R}^{3}$ is the error posture.

Remark 1: It should be noted that the vehicle-to-vehicle distance and the relative inter-vehicle orientation $e_{\theta}$ are measured by sensors that monitor the rear end of the lead vehicle. Since we know the position and the orientation of the sensors and the frame $R_{2 f} x y$ relative to the following vehicle frame $A_{2} x_{A 2} y_{A 2}$, as well as the position and the orientation of $R_{I r} x y$ with respect to $A_{l} x_{l} y_{A l}$ using geometric argument, we can calculate the position $\left(e_{x}, e_{y}\right)$ and orientation $e_{\theta}$ of the frame $R_{2 f}$ with respect to the coordinate frame $R_{l r}$.

Differentiating (18) with respect to time and taking into account Eqs. (4) and (5), after some work, the inter-vehicle kinematics in error coordinates is obtained as

$$
\begin{aligned}
& {\left[\begin{array}{l}
\dot{e}_{x} \\
\dot{e}_{y} \\
\dot{e}_{\theta}
\end{array}\right]=\left[\begin{array}{cc}
\cos e_{\theta} & -L_{2} \sin e_{\theta} \\
\sin e_{\theta} & L_{2} \cos e_{\theta} \\
0 & 1
\end{array}\right]\left[\begin{array}{c}
A_{2} v_{A_{2} x_{A_{2}}} \\
\omega_{2}
\end{array}\right]-} \\
& -\left[\begin{array}{cc}
1 & 0 \\
0 & -L_{1} \\
0 & 1
\end{array}\right]\left[\begin{array}{c}
{ }^{A_{1}} v_{A_{1} x_{A_{1}}} \\
\omega_{1}
\end{array}\right]+\dot{\theta}_{2}\left[\begin{array}{ccc}
0 & 1 & 0 \\
-1 & 0 & 0 \\
0 & 0 & 0
\end{array}\right]\left[\begin{array}{c}
e_{x} \\
e_{y} \\
e_{\theta}
\end{array}\right]
\end{aligned}
$$

where $\left({ }^{A_{1}} v_{A_{1} x_{A}}, \omega_{1}\right)$ and $\left({ }^{A_{2}} v_{A_{2} x_{A 2}}, \omega_{2}\right)$ are the linear and angular velocities of the lead and following vehicle, respectively. From now on, to simplify the notations, the vehicle linear velocities will be denoted as follows: $v_{A_{1}}:={ }^{A_{1}} v_{A_{1} x_{A_{1}}}$ and $v_{A_{2}}:={ }^{A_{2}} v_{A_{2} x_{A_{2}}}$ for the lead and the following vehicle, respectively. The linear and angular velocities of the following vehicle are the control inputs of the system (20). 


\section{PROBLEM FORMULATION}

One of the challenges in designing controllers for automatic vehicle tracking is to decide on the desired trajectory of the following vehicle. On a curved road section, traveling an arc concentric to that traveled by the lead car but with bigger or smaller radius may be unacceptable from a practical point of view, (for example, the problem of "cutting the corner") (Fig. 3).

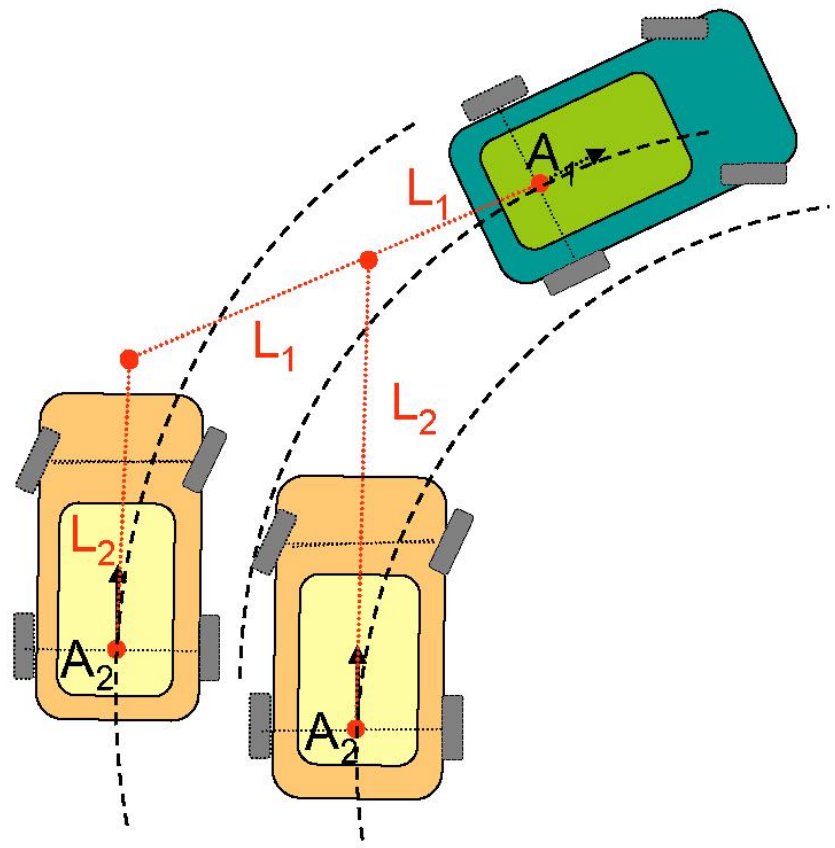

Fig. (3). Circular motion with $L_{1} \neq L_{2}$.

Assuming constant velocity maneuvers for forward driving of the lead vehicle, we are interested in a tracking scenario where the two-vehicle convoy will travel concentric arcs of same radii, (Fig. 4), with prescribed inter-vehicle distance depending on the curvature radius of the path taken by the lead vehicle and an a priori known desired intervehicle spacing, which is defined for the case of straight line driving.

In the case of straight line motion of the lead vehicle, (Fig. 5), the desire inter-vehicle distance is defined to be:

$d_{\text {des }}=S_{1} P_{2}=2 L-\left(A_{1} S_{1}+P_{2} B_{2}+l_{2}\right)$

where $L=L_{1}=L_{2}$ is a known constant distance determined from consideration of safety driving, sensor requirements, look-ahead visibility during the turning maneuver.

At steady state, the inter-vehicle distance during circular motion of constant radius is a little smaller, and has different value for different curvature radius of the leader path, and in that way, the requirement of circular motion of same radii is satisfied.

Using trigonometric argument, the inter-vehicle distance $S_{1} P_{2}$ at steady state is obtained as follows

$S_{1} P_{2}=\operatorname{sqrt}\left[2 L^{2}-2 L b+b^{2}+2(l-b) L \cos e_{\theta s s}\right]$ where $b=l_{2}+B_{2} P_{2}$. For rectilinear motion of the lead vehicle et steady state, the inter-vehicle orientation is $e_{\theta s s}=0$, and for the inter-vehicle distance $S_{1} P_{2}$ the expression (21) follows readily.

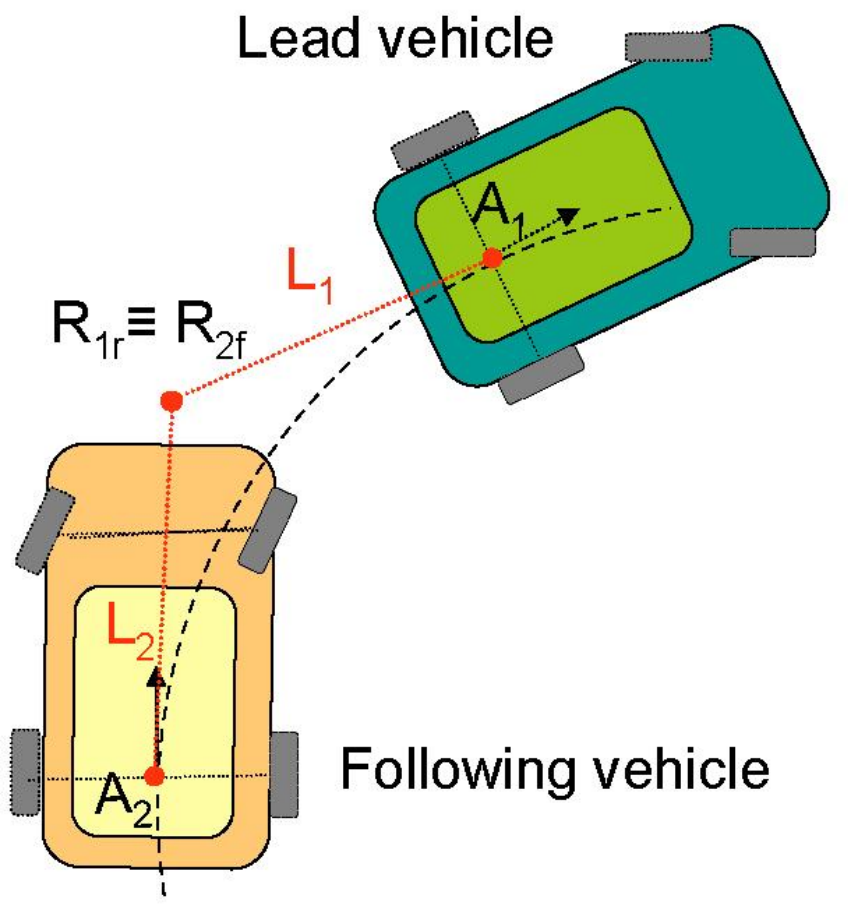

Fig. (4). Circular motion with $L_{1}=L_{2}$.

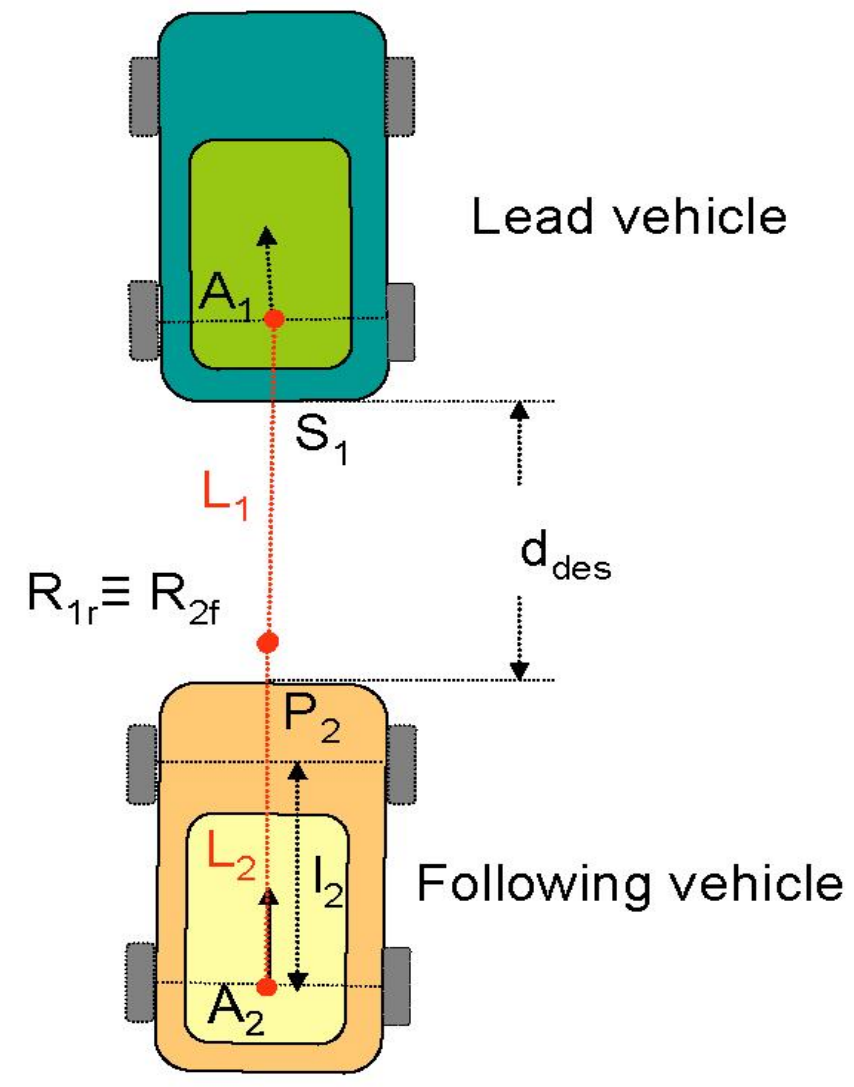

Fig. (5). Desired inter-vehicle spacing. 
Given the inter-vehicle kinematics in error coordinates (20), and assuming that the linear and angular velocities of the lead vehicle $\left(v_{A 1}, \omega_{l}\right)$ are unknown constant parameters and are not available for feedback control, the control objective is to asymptotically track the virtual reference point $R_{I r}$ the lead vehicle with the reference point $R_{2 f}$ of the following vehicle.

\section{ADAPTIVE CONTROL DESIGN}

In this paper, we consider the problem of controlling the motion of the following vehicle during constant velocity maneuvers for forward driving of the lead vehicle. Since the leader velocities are not available for feedback control design, we propose an adaptive control law, which achieves asymptotic stabilization of the closed-loop system in error coordinates. The control velocities of the following vehicle are computed using the leader velocity estimates $\left(\hat{v}_{A 1}, \hat{\omega}_{1}\right)$ obtained from the dynamic (adaptive) part of the proposed controller. For this purpose, let us consider the following change of inputs in (20)

$\left[\begin{array}{l}u_{1} \\ u_{2}\end{array}\right]=\left[\begin{array}{cc}\cos e_{\theta} & -L_{2} \sin e_{\theta} \\ \sin e_{\theta} & L_{2} \cos e_{\theta}\end{array}\right]\left[\begin{array}{c}v_{A 2} \\ \omega_{2}\end{array}\right]$.

One easy verifies that the transformation matrix in (23) is nonsingular when $L_{2} \neq 0$.

Using the input transformations (23), the inter-vehicle kinematic equations in error coordinates (20) can be written in the form

$\dot{e}_{x}=-v_{A_{1}}+\omega_{1} e_{y}+u_{1}$

$\dot{e}_{y}=\left(L_{1}-e_{x}\right) \omega_{1}+u_{2}$

$e_{\theta}=-\omega_{1}+\left(-u_{1} \frac{\sin e_{\theta}}{L_{2}}+u_{2} \frac{\cos e_{\theta}}{L_{2}}\right)$

The adaptive control design is based on a reduced-order system composed of the first two equations of (24)

$\dot{e}_{x}=-v_{A_{1}}+\omega_{1} e_{y}+u_{1}$

$\dot{e}_{y}=\left(L_{1}-e_{x}\right) \omega_{1}+u_{2}$

Consider the subsystem (25) and assume that the leader velocities $v_{A l}=$ cte $>0$ and $\omega_{1}=c t e$, are unknown constant parameters. The control problem consists in finding an adaptive feedback control law for the system (25) with inputs $\left(u_{1}, u_{2}\right)$ such that

$\lim _{t \rightarrow \infty}\left(e_{x}(t)\right)=0$ and $\lim _{t \rightarrow \infty}\left(e_{y}(t)\right)=0$.

Assume that $L_{2} \neq 0$ and consider the control

$u_{1}=-k_{x} e_{x}+\hat{v}_{A_{1}}-\widehat{\omega}_{1} e_{y}$

$u_{2}=-k_{y} e_{y}-\left(L_{1}-e_{x}\right) \hat{\omega}_{R}$

where $k_{x}$ and $k_{y}$ are positive gains. We consider the following Lyapunov function candidate

$V=\frac{1}{2} e_{x}^{2}+\frac{1}{2} e_{y}^{2}+\frac{1}{2 \gamma_{v}} \tilde{v}_{A_{1}}^{2}+\frac{1}{2 \gamma_{\omega}} \tilde{\omega}_{1}^{2}$ where $\tilde{v}_{A 1}$ and $\tilde{\omega}_{1}$ are the parameter errors

$\tilde{v}_{A_{1}}=\hat{v}_{A_{1}}-v_{A_{1}}$

$\tilde{\omega}_{1}=\hat{\omega}_{1}-\omega_{1}$

and $\gamma_{v}=$ cte $>0$, and $\gamma_{\omega}=$ cte $>0$ are the adaptation gains. Using (29), the derivative of $V$ is obtained in the form

$$
\begin{aligned}
& \dot{V}=-k_{x} e_{x}^{2}-k_{y} e_{y}^{2}+\tilde{v}_{A_{1}}\left(e_{x}+\frac{1}{\gamma_{v}} \dot{\tilde{v}}_{A_{1}}\right)+ \\
& +\tilde{\omega}_{1}\left[-e_{x} e_{y}-e_{y}\left(L_{1}-e_{x}\right)+\frac{1}{\gamma_{\omega}} \dot{\tilde{\omega}}_{R}\right]
\end{aligned}
$$

where all the terms containing $\widetilde{v}_{A 1}$, as well as $\widetilde{\omega}_{1}$, have been grouped together. To eliminate them, the update law is chosen as

$\dot{\hat{v}}_{A_{1}}=-\gamma_{v} e_{x}$

$\dot{\hat{\omega}}_{1}=\gamma_{\omega} L_{1} e_{y}$

and the derivative of $V$ is obtained as

$\dot{V}=-k_{1} e_{x}^{2}-c_{1} e_{y}^{2} \leq 0$.

The resulting closed-loop adaptive system in error coordinates becomes

$\dot{e}_{x}=-k_{x} e_{x}+\tilde{v}_{A_{1}}-e_{y} \tilde{\omega}_{1}$

$\dot{e}_{y}=-k_{y} e_{y}-\left(L_{1}-e_{x}\right) \tilde{\omega}_{1}$

$\dot{\tilde{v}}_{R x}=-\gamma_{v} e_{x}$

$\dot{\tilde{\omega}}=-\gamma_{\omega} L_{1} e_{y}$

Proposition 1: Assume that lead vehicle linear and angular velocities $\left(v_{A l}, \omega_{l}\right)$ are bounded unknown constant parameters, $v_{A l}>c_{v}=c t e>0$ and $L_{1} \neq 0$. If the control law given by (27) is applied to (25) where the lead vehicle velocity estimates $\left(\hat{v}_{A 1}, \hat{\omega}_{1}\right)$ are obtained from the parameter update law (31), the origin $x=\left[e_{x}, e_{y}, \tilde{v}_{A 1}, \tilde{\omega}_{1}\right]^{T}=0$ of the closed-loop system in error coordinates (33) is asymptotically stable.

Proof. The system has an equilibrium point at the origin. The function (28) is continuously differentiable and positive definite. From (32), it follows that (28) is non-increasing, $(V(t) \leq V(0))$, and this in turn implies that $e_{x}(t), e_{y}(t), \tilde{v}_{A 1}(t)$ and $\tilde{\omega}_{1}(t)$ are uniformly bounded with respect to the initial conditions. By application of the LaSalle-Yoshizawa Theorem [17, p.24, Theorem 2.1],

$\lim _{t \rightarrow \infty} \dot{V}=-k_{1} e_{x}^{2}-c_{1} e_{y}^{2}=0$.

Therefore

$e_{x}(t) \rightarrow 0, e_{y}(t) \rightarrow 0$

as $t \rightarrow \infty$. From the third and forth equations of (33), it follows that $\dot{\tilde{v}}_{R x} \rightarrow 0, \dot{\tilde{\omega}}_{R} \rightarrow 0$ as $t \rightarrow \infty$.

The first equation of (33) can be seen as a derivative of a differentiable function $e_{x}(t)$ from $R^{+}$to $R$, which converges to 
limit value (0) when $t$ tends to infinity, and its derivative, (the right side of this equation) can be seen as a sum of two terms, one, $\left(\tilde{v}_{A 1}\right)$, being uniformly continuous since its derivative is bounded, and the other, $\left(-k_{x} e_{x}-e_{y} \tilde{\omega}_{1}\right)$, which tends to zero. By application of the extended version of Barbalat's Lemma [18], it follows that $\dot{e}_{x}(t) \rightarrow 0$ as $t \rightarrow \infty$, and this in turn implies that the term

$\tilde{v}_{R x} \rightarrow 0$

as $t \rightarrow \infty$.

In the same fashion, it can be proved from the second equation of (33) that $\dot{e}_{y}(t) \rightarrow 0$ as $t \rightarrow \infty$ and this in turn implies that the term

$\tilde{\omega}_{R} \rightarrow 0$.

Since the dynamics of $e_{\theta}$ has not been taken into account into the feedback control design, the next step in the stability analysis is to establish asymptotic convergence of $e_{\theta}$ to the equilibrium state $e_{\theta s s}$ which depends of lead vehicle linear and angular velocities $\left(v_{A l}, \omega_{1}\right)$, or equivalently the curvature radius of the path driven by the lead vehicle $\rho \kappa=v_{A I} / \omega_{1}$, and the distances $L_{1}$ and $L_{2}$. We analyze the zero dynamics of $e_{\theta}$ assuming that $e_{x}(t) \equiv 0 ; e_{y}(t) \equiv 0$ and $\tilde{v}_{A 1} \equiv 0 ; \tilde{\omega}_{1} \equiv 0$ for all time. In this case, substituting (27) into the third equation of (24) after some work, the zero dynamics of $e_{\theta}$ is obtained as

$\dot{e}_{\theta}=-\frac{v_{A_{1}}}{L_{2}} \sin e_{\theta}-\omega_{1}\left(1+\frac{L_{1}}{L_{2}}\right) \cos e_{\theta}$.

Since we are interested in tracking scenario where at steady state the vehicle convoy will travel concentric arcs of same radii, we assume that $L_{1}=L_{2}=L$. Using the transformation

$\Phi:=\tan \left(e_{\theta} / 2\right)$

equation (38) can be rewritten in the form

$\dot{\Phi}=-\frac{v_{A_{1}}}{L} \Phi-\omega_{1}$.

Equation (40) represents first order linear differential equation with constant coefficients. The solution of (41) with initial condition $\Phi(0)=0$ is given in the form

$\Phi(t)=\frac{L \omega_{1}}{v_{A_{1}}}\left[\exp \left(-\frac{v_{A_{1}}}{L} t\right)-1\right]$

From (41), it can be seen that

$\Phi(\infty)=-\frac{L \omega_{1}}{v_{A_{1}}}=\Phi_{s s}=$ cte .

Using the inverse transformation of (39), the relative orientation of the vehicles at steady state $e_{\theta s s}$ is obtained as

$e_{\theta s s}=2 a \tan \left(l / \rho_{R}\right)=c t e$

where
$\rho_{R}=v_{A_{1}} / \omega_{1}=c t e$

is the curvature radius of the path travel by the lead vehicle. Since $e_{x}=e_{y}=0$, i.e., the reference points $R_{l r}$ and $R_{2 f}$ coincide, and $L_{1}=L_{2}=L$, using geometrical argument, it follows that the two vehicles will travel concentric arcs of same radii, which complete the proof.

Using the inverse transformations of (23) and expressions (27) for the control inputs $\left(u_{1} u_{2}\right)$, we obtain expressions for the actual control inputs $\left(v_{A 2}, \omega_{2}\right)$ and in turn, from (18), for the front wheel steering angle $\alpha_{2}$ of the following vehicle.

\section{V.SIMULATION RESULTS}

To illustrate the effectiveness of the proposed controller, several simulations are carried out in order to evaluate the vehicle behavior and tracking accuracy. In the simulation using MATLAB, a planar bicycle 2DOFs vehicle model was used. The longitudinal vehicle base was chosen to be $l_{1}=l_{2}=1$ $=2 \mathrm{~m}$. For simplicity in the simulation, we set $1=\mathrm{P}_{2} \mathrm{~A}_{2}$ and $A_{1} S_{1}=0$, (Fig. 5). The distance $L=L_{i}=L_{2}$ was chosen to be $\mathrm{L}=4 \mathrm{~m}$, which corresponds to desired inter-vehicle separation $\mathrm{d}_{\mathrm{des}}=6 \mathrm{~m}$ in the case of straight line motion of the lead vehicle, (Fig. 6). The gains in the control law (27) were chosen as $\mathrm{k}_{\mathrm{x}}=8 ; \mathrm{k}_{\mathrm{y}}=20 ; \gamma_{\mathrm{v}}=5 ; \gamma_{\mathrm{m}}=0.5$. The initial position and orientation of the following vehicle in the inertial frame Fxy were chosen to be ${ }^{\mathrm{F}} \mathrm{x}_{\mathrm{A} 2}(0)=0 \mathrm{~m},{ }^{\mathrm{F}} \mathrm{y}_{\mathrm{A} 2}(0)=0 \mathrm{~m}$, $\theta_{2}(0)=0 \mathrm{rad}$. The initial position and orientation of the lead vehicle in the inertial frame Fxy were chosen to be $^{F} x_{A_{1}}(0)=9.3 m,{ }^{F} y_{A_{1}}(0)=0, \theta_{1}(0)=-0,25 \mathrm{rad}$. In this case, the initial inter-vehicle distance $\mathrm{S}_{1} \mathrm{P}_{2}=7.37 \mathrm{~m}$ which is different from $d_{\text {des }}$. The initial values of the error coordinates were $e_{x}(0)=1 \mathrm{~m} ; \mathrm{e}_{\mathrm{y}}(0)=-1 \mathrm{~m} ; \mathrm{e}_{\theta}(0)=-0.25 \mathrm{rad}$. The initial estimates for the leader linear and angular velocities were chosen to be $\hat{v}_{A 1}(0)=2 \mathrm{~m} / \mathrm{s}$ and $\hat{\omega}_{1}(0)=0 \mathrm{rad} / \mathrm{s}$, and are different from the real values of the lead vehicle velocities $\left(\mathrm{v}_{\mathrm{A} 1}=4 \mathrm{~m} / \mathrm{s}, \omega_{1}=0.27 \mathrm{rad} / \mathrm{s}\right.$ ) for the first maneuver (Table 1). The leader path consists of three consecutive constant velocity maneuvers: turning both to the left and to the right followed by a straight line motion. The corresponding actual lead vehicle velocities are given in Table $\mathbf{1}$.

In the first simulation, from Fig. (6), we can see the planar paths drown by the vehicle guide points $A_{1}$ and $A_{2}$ of the lead and following vehicle, respectively, (the mid-points of the rear vehicle axles). At steady state, the following vehicle tracks the path taken by the lead vehicle without lateral error.

From Fig. (7), we can see the evolution in time of the error coordinates. For each maneuver, the position errors $e_{x}(t)$ and $e_{y}(t)$ tend asymptotically to zero, i.e., at steady state, the look-ahead virtual point $R_{2 f}$ coincides with the virtual reference point $R_{l r}$. The inter-vehicle orientation $e_{\theta}(t)$ tends also asymptotically to a constant value $e_{\theta s s}$.

As shown in Fig. (8), the estimates of the leader velocities tend asymptotically to their actual values.

From Fig. (9), we can see the evolution in time of the inter-vehicle distance $S_{l} P_{2}$. The steady-state inter-vehicle 
Table 1.

\begin{tabular}{|c|c|c|c|c|}
\hline & Radius of Turn $\boldsymbol{\rho}_{\boldsymbol{R}}[\mathbf{m}]$ & Duration $[\mathbf{s}]$ & Leader Linear Velocity $\boldsymbol{v}_{\boldsymbol{A} \boldsymbol{I}}[\mathbf{m} / \mathbf{s}]$ & Leader Angular Velocity $\boldsymbol{\omega}_{\boldsymbol{I}}[\mathbf{r a d} / \mathbf{s}]$ \\
\hline \hline Maneuver 1 - left turn & 15 & 10 & 4 & 0.27 \\
\hline Maneuver 2 - right turn & 10 & 22 & 2 & -0.2 \\
\hline Maneuver 3 - straight line & - & 8 & 5 & 0 \\
\hline
\end{tabular}

distance during circular motion of constant radius is different for different values of the curvature radius of the leader path For example, for the first maneuver $\left(\rho_{R}=15 \mathrm{~m}\right)$, at steady state $S_{1} P_{2}=5.82 \mathrm{~m}$; for the second maneuver $\left(\rho_{R}=10 \mathrm{~m}\right)$, at steady state $S_{1} P_{2}=5.62 \mathrm{~m}$ and are a little smaller compared to the desired distance $d_{d e s}$ of $6 m$ during the straight line motion. In that way, the requirement of circular motion of same radii of the vehicles is satisfied. This is in accordance with the theoretical result obtained in Section IV.

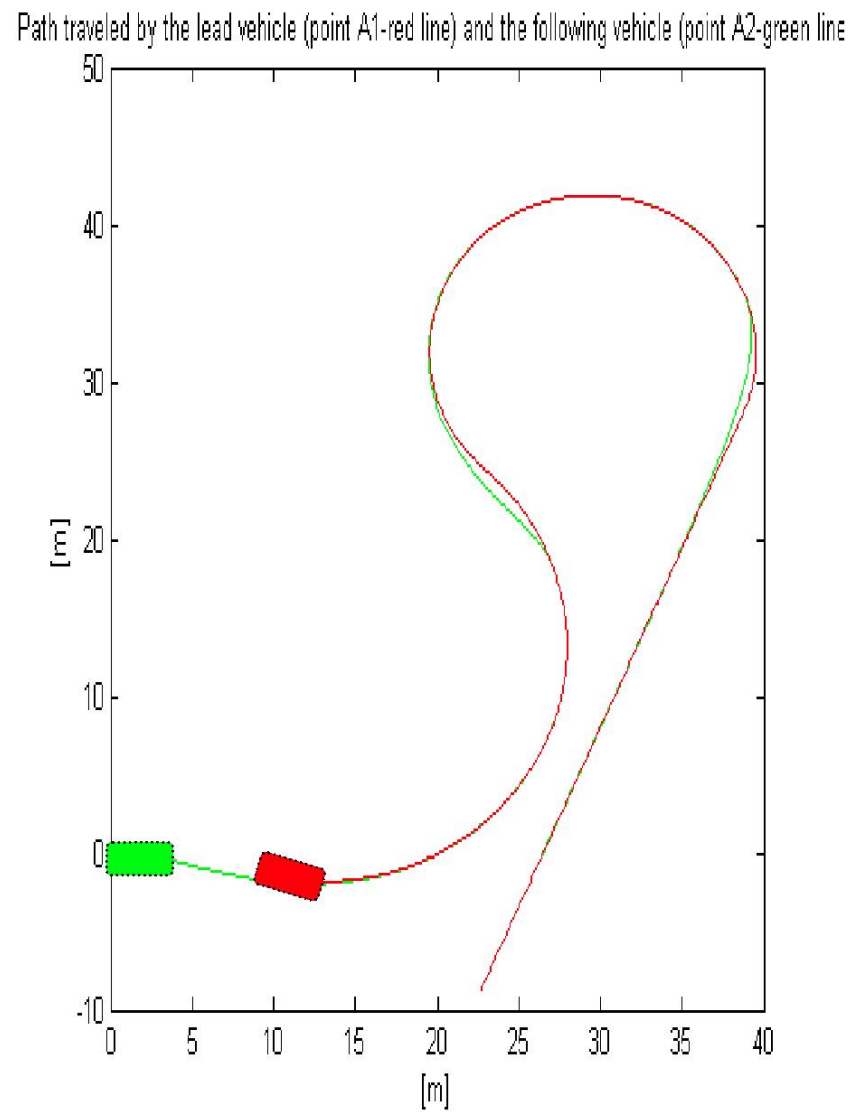

Fig. (6). A planar path drown by the vehicle guide points (the midpoints of the rear vehicle axles); red line (point $A_{l}$, lead vehicle), green line (point $A_{2}$, following vehicle), $L_{1}=L_{2}=4 \mathrm{~m} ; d_{d e s}=6 \mathrm{~m}$.

The results of the simulation verify the validity of the proposed controller.

\section{CONCLUSION}

This paper considers the problems of kinematic modeling of a two-vehicle convoy and the design of a vehicle following controller that tracks the trajectory of the vehicle head with prescribed inter-vehicle distance. It is shown that

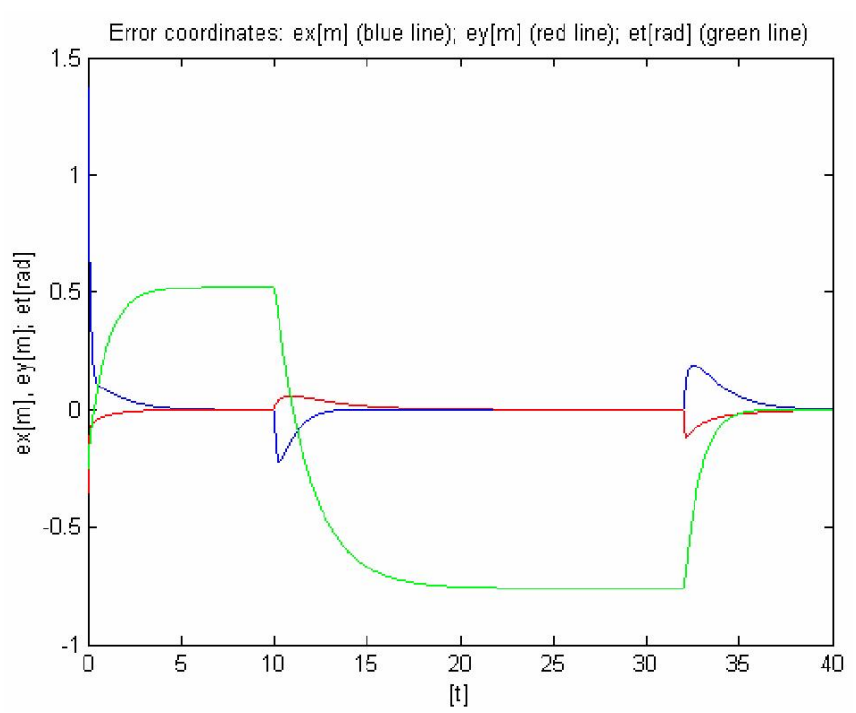

Fig. (7). Evolution of the error coordinates in time: $e_{x}(t)$ (blue line); $e_{y}(t)$ (red line); $e_{\theta}(t)$ (green line); $L_{1}=L_{2}=4 \mathrm{~m} ; d_{\text {des }}=6 \mathrm{~m}$.

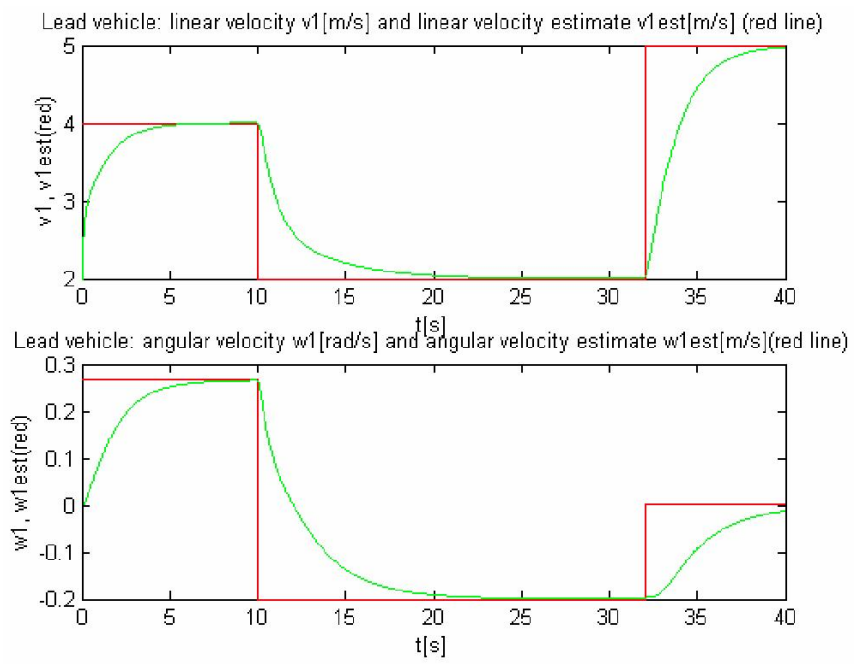

Fig. (8). Evolution in time of the lead vehicle velocities estimates $\left(\hat{v}_{A 1}, \hat{\omega}_{1}\right)$ (green line); lead vehicle actual velocities $v_{A l}$ and $\omega_{I}$ (red line); $L_{l}=L_{2}=4 m ; d_{d e s}=6 m$.

the use of standard robotic methodology by using homogeneous transformation matrices can be used effectively for modeling the dynamics of the system. In this paper, a systematic method is developed for the design of an adaptive tracking controller for vehicle following. The control problem is solved alike trajectory tracking by coupling lateral and longitudinal control. A specific feature of the control scheme is that it allows the vehicle to follow the car instead of the road, i.e., a strategy for autonomous 


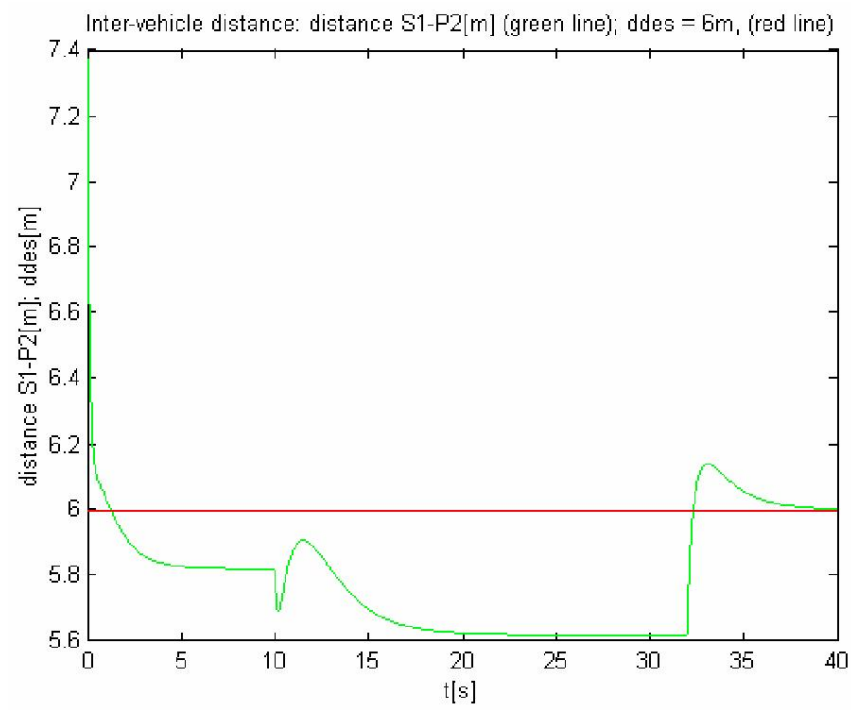

Fig. (9). Evolution of the inter-vehicle distance $S_{1} P_{2}$ time (green line); Desired inter-vehicle distance during straight line motion of the leader: $d_{d e s}=6 m$ (red line); $L_{l}=L_{2}=4 m$.

control was presented without the use of road infrastructure or inter-vehicle communication. The linear and angular velocities of the lead vehicle are unknown constant parameters, and the curvature radius of the lead vehicle path is also unknown. The proposed adaptive control law achieves asymptotic stabilization of the closed-loop system in error coordinates. Assuming constant velocity maneuvers of the leader, at steady state, the following vehicle will track the path taken by the lead vehicle. In that way, the problem of "cutting the corner" has been overcome. The results of the simulation verify the validity of the proposed controller. Future research will address the problem of a unified approach to control for backward and forward driving of an autonomous convoy with time-varying velocities.

\section{REFERENCES}

[1] K. Cheok, G. Smidt, K. Kobayashi, J. Overholt, P. Lescoe, "A fuzzy logic intelligent control system paradigm for an in-line-ofsight leader-following $R M M W V^{\prime \prime}$, Journal of Robotic Systems, vol. 14, no. 6, pp. 407-420, 1997.

[2] B. Brumitt, M. Hebert, "Experiments in autonomous Driving with concurrent goals and multiple vehicles", in Proceedings of IEEE International Conference on Robotics and Automation, 1998, pp. 1895-1902.
[3] R. Hogg, A. Rankin, M. McHenry, D. Helmick, C. Bergh, S. Roumeliotis, L. Matthies, " Algoritms and sensors for small robot path following", in Proceedings of IEEE International Conference on Robotics and Automation, 2002, pp. 3850-3857.

[4] H. Fritz, "Longitudinal and lateral control of heavy duty trucks for automated vehicle following in mixed traffic: experimental results from the CHAUFFEUR project", in Proceedings of International Conference on Control Applications, 1999, pp. 1348-1352.

[5] R. White, M. Tomizuka, "Autonomous following lateral control of heavy vehicles using laser scanning radar", in Proceedings of American Control Conference, 2001, pp. 2333-2337.

[6] G. Lu, J. Huang, M. Tomizuka, "Vehicle lateral control under fault in front and/or rear sensors: final report', Research Report, UCBITS- PRR-2004-36, 2004.

[7] A. Ferrara, R. Librino, A. Massola, M. Miglietta, C. Vecchio, "Sliding mode control for urban vehicle platooning", in Proceedings of IEEE Intelligent Vehicle Symposium, 2008, pp. 877-882.

[8] P. Petrov, M. Parent, "An Adaptive Tracking Controller for Backward Driving of a Two-Vehicle Convoy", in Proc. $9^{\text {th }}$ IEEE Intertnational Conference on Intelligent Transportation Systems, 2006, pp.1376-1381.

[9] D. Yanakievi, I. Kanellakopoulos, "Nonlinear spacing policies for automated heavy-duty vehicles", IEEE Transaction on Vehicular Technology, vol. 47, no. 4, pp. 1365-1377, Nov. 1998.

[10] S. Shladover, C. Desoer, J. Hedrik, M. Tomizuka, J. Warland, "Automatic vehicle control developments in the PATH program", IEEE Transaction on Vehicular Technology, vol. 40, no. 1, pp. 114130, 1991.

[11] T. Fujioka, M. Omae, "Vehicle following control in lateral direction for platooning", Vehicle System Supplement, vol. 28, pp 422-437, 1998.

[12] P. Petrov, O. Boumbarov, "Nonlinear Adaptive Control of a TwoVehicle Autonomous Convoy Using a Look-Ahead Approach", in Proc. 7* WSEAS International Conference on Signal Processing, Robotics and Automation, 2008, pp. 55-60.

[13] M. Pham, D. Wang, "A Unified nonlinear controller for a platoon of car-like vehicles", in Proceedings of American Control Conference, 2004, pp. 2350- 2355.

[14] J. Bom, B. Thuilot, F. Marmoiton, P. Martinet, "Nonlinear control for urban vehicles platooning, relying upon a kinematic kinematic GPS", in Proceedings of IEEE International Conference on Robotics and Automation, 2005, pp. 4149-4154.

[15] P. Petrov, J. de Lafontaine, M. Tetreault, "Hybrid Feedback Control for the Parking Problem of a Load-Haul-Dump Mine Vehicle", in Proceedings of IEEE/RSJ International Conference on Intelligent and Robotics System, 1998, pp. 1907-1912.

[16] M. Spong, M. Vidyasagar, Robot dynamics and control, John Wiley\&Sons, 1988.

[17] M. Kristic, I. Kanellakopoulos, P. Kokotovic, Nonlinear and Adaptive Control Design, John Wiley\&Sons, New York, 1995.

[18] C. Samson, "Control of chained systems: Application to path following and time-varying point stabilization of mobile robots", IEEE Transactions on Automation Control, vol. 40, no. 1, pp.64-75, 1995. 